\title{
Improving Revealed Preference Bounds on Demand Responses
}

\author{
Richard Blundell \\ University College London and IFS \\ Martin Browning \\ University of Oxford \\ Ian Crawford \\ University of Surrey and IFS \\ Feburary 2007
}

\begin{abstract}
There are three key dimensions by which revealed preference bounds on consumer demand responses to price variation can be improved. The first relates to the improvements that arise from using expansion paths for given relative prices, E-bounds. The second concerns the addition of new price information. Thirdly, there are improvements due to assuming separability. Our previous research has examined the first two cases. In this paper we show how to impose these separability assumptions within a fully nonparametric analysis and distinguish between weak and homothetic separability. We also apply these ideas to the analysis of demand responses using UK household level data.
\end{abstract}

Key Words: Demand responses, revealed preference, relative prices, separability, semiparametric regression.

JEL Classification: D12, C14, C43.

Acknowledgements: Revised version of a paper presented at the Conference to honor Dan McFadden, Berkeley, May 2005. We would like to thank the editor and two annonymous referees for their helpful comments. The research is part of the program of research of the ESRC Centre for the Microeconomic Analysis of Public Policy at IFS. Funding from the ESRC, grant number R000239865, the Leverhulme Trust and from the Danish National Research Foundation through its grant to CAM is gratefully acknowledged. Material from the FES made available by the ONS through the ESRC Data Archive has been used by permission of the controller of HMSO. Neither the ONS nor the ESRC Data Archive bear responsibility for the analysis or the interpretation of the data reported here. The usual disclaimer applies. 


\section{Introduction}

Measuring the responses of consumers to variation in relative prices and in income is at the centre of applied welfare economics: it is a vital ingredient of tax policy reform analysis and is also key to the measurement of market power in modern empirical industrial economics. Parametric models have dominated applications in this field but this is increasingly seen to be both unwise and unnecessary. To quote Dan McFadden in his presidential address to the Econometric Society: "[parametric regression] interposes an untidy veil between econometric analysis and the propositions of economic theory". Popular parametric models place strong assumptions on both income and price responses. The objective of the research agenda which underlies this paper is to accomplish all that is required from parametric models of consumer behaviour using only nonparametric regression and revealed preference theory.

The aim of this research is to combine the inequalities which summarise the axioms of revealed preference (RP) with the nonparametric estimation of consumer expansion paths (Engel curves). The empirical setting we have in mind is one where we have consumer level data on commodity expenditures and incomes across a finite set of discrete relative price or tax regimes. In Blundell, Browning and Crawford $(2003,2007)$ we have shown how to use the RP inequalities of Afriat (1967) and Varian $(1982,1983)$ in such an empirical setting to generate tight bounds on welfare measures and on consumers demand responses. In this paper we extend these results to examine the impact of nonparametric separability restrictions. These are shown to further tighten the bounds on demand responses. However, without parametric restrictions demand responses remain only set identified in the sense of Manski (2003).

Freeing up the variation in demand responses to relative price changes across the income distribution is one of the key contributions of this research agenda. Historically parametric specifications in the analysis of consumer behavior have been based on the Working-Leser or Piglog form of preferences that underlie the popular Almost Ideal and Translog demand models of Deaton and Muellbauer (1980) and Jorgenson, Lau and Stoker (1982). Even though more recent empirical studies have suggested further nonlinear income terms, (see, for example, Hausman, Newey, Ichimura and Powell (1995), Lewbel (1991), Blundell, Pashardes and Weber (1993), Banks, Blundell and Lewbel (1997)), responses to relative prices at different 
incomes for these parametric forms remain unnecessarily constrained.

There are three broad mechanisms for the improvement of revealed preference bounds. The first of these uses nonparametrically estimated Engel curves (or expansion paths) to provide tight bounds on cost of living indices and on demand responses. These 'expansion path based bounds' are labelled E-bounds, see Blundell, Browning and Crawford (2003, 2007). The second improvement comes from the use of more price information. Typically consumers are observed choosing demands under a finite set of different relative prices. Relative prices are assumed to be different over time or across different markets as supply costs and production technology changes. Blundell, Browning and Crawford (2007) investigate the value of more relative price variation in improving the bounds on demand responses. In this paper, we turn to improvements that can be achieved through grouping goods according to separability. We show how to impose separability restrictions within a fully nonparametric analysis and distinguish between weak, homothetic and exact index separability. These improvements are first considered theoretically using standard consumer theory arguments and are then applied to the empirical analysis of consumer behaviour.

In our empirical analysis relative price variation occurs over time and we consider consumer behaviour as it is recorded in standard repeated consumer expenditure surveys such as the US Consumers Expenditure Survey and the UK Family Expenditure Survey. The latter is the source for our empirical analysis. We observe samples of consumers, each of a particular household type, at specific points in time. Assuming consumers are price-takers, we can recover expansion paths by estimating Engel curves at each point in time. We present E-bounds for own and cross price responses using these expansion paths. We allow for sampling variation in the estimated expansion paths and consider whether perturbations to preferences can be found that allow revealed preference theory to be maintained while lying within standard confidence intervals.

The remainder of the paper is as follows: in section 2 we examine the improvements in RP bounds on demand responses that occur due to the use of expansion paths. We also consider the advantage of adding in more relative price variation. This section draws heavily on Blundell et al (2007). In Section 3 we consider improvements that can result 
from different forms of separability. We show how to impose separability restrictions within a fully nonparametric analysis and distinguish between weak, homothetic and exact index separability. These improvements are first considered theoretically using standard consumer theory arguments and are then applied to the empirical analysis of consumer behaviour. In section Section 4 we apply these ideas to analyse demand responses using the household level data in the UK Family Expenditure Survey. Section 5 concludes.

\section{Improving Bounds with Expansion Paths}

Let $\mathbf{p}$ be a $J$-vector of prices and $x$ total expenditure. We assume that every agent responds to a given budget $(\mathbf{p}, x)$, with a unique, positive demand $J$-vector which we denote $\mathbf{q}(\mathbf{p}, x)$. We further assume that these demands satisfy adding-up $\left(\mathbf{p}^{\prime} \mathbf{q}(\mathbf{p}, x)=x\right)$ and normality $\left(\mathbf{q}\left(\mathbf{p}, x^{\prime}\right) \geq \mathbf{q}\left(\mathbf{p}, x^{\prime \prime}\right)\right.$ if $\left.x^{\prime}>x^{\prime \prime}\right)$. We shall only be considering demand functions for a finite number of distinct relative prices; an expansion path for for a given price vector $\mathbf{p}_{t}$ is defined as $\mathbf{q}_{t}(x)=\mathbf{q}\left(\mathbf{p}_{t}, x\right)$ (with $q_{t}^{j}$ denoting the demand for good $j$ in period $t$ ). In our empirical analysis below we shall estimate an expansion path for each price regime in our data; we refer to these expansion paths as observed expansion paths or observed demands.

The goal of our research is to find the set of possible demand responses to a new budget and set of relative prices, such that these are consistent with already observed demands and utility maximisation. That is, we take a set of observed prices and expansion paths $\left\{\mathbf{p}_{t}, \mathbf{q}_{t}(x)\right\}_{t=1, . . T}$ and ask what demands that are consistent with utility theory could arise, given a new budget $\left\{\mathbf{p}_{0}, x_{0}\right\}$. To do this with no appeal to parametric assumptions we use revealed preference conditions. Specifically, we take the Strong Axiom of Revealed Preference (SARP) (see Varian (1982) for the relationship between SARP and the more usual GARP). To state SARP we need to define what we mean by revealed preference. If at prices $\mathbf{p}_{t}$ the agent chooses $\mathbf{q}_{t}$ and we have $\mathbf{p}_{t}^{\prime} \mathbf{q}_{t} \geq \mathbf{p}_{t}^{\prime} \mathbf{q}_{s}$ then we say that $\mathbf{q}_{t}$ is directly revealed weakly preferred to $\mathbf{q}_{s}$, denoted $\mathbf{q}_{t} R^{0} \mathbf{q}_{s}$. If we have a chain $\mathbf{q}_{t} R^{0} \mathbf{q}_{u}, \mathbf{q}_{u} R^{0} \mathbf{q}_{v}, \ldots \mathbf{q}_{w} R^{0} \mathbf{q}_{s}$ then we say that $\mathbf{q}_{t}$ is revealed weakly preferred to $\mathbf{q}_{s}$ and denote this by $\mathbf{q}_{t} R \mathbf{q}_{s}$. Given this, SARP is defined by the following:

Definition 1 SARP: $\mathbf{q}_{t} R \mathbf{q}_{s}$ and $\mathbf{q}_{t} \neq \mathbf{q}_{s}$ implies not $\mathbf{q}_{s} R^{0} \mathbf{q}_{t}$ (that is, $\left.\mathbf{p}_{s}^{\prime} \mathbf{q}_{s}<\mathbf{p}_{s}^{\prime} \mathbf{q}_{t}\right)$ for all $s, t$. 
The main features of our analysis can be captured in a simple two good, two period example, as shown in Figure $1 .^{1}$ In this figure, the two expansion paths are shown as $\mathbf{q}_{1}(x)$ and $\mathbf{q}_{2}(x)$. The hypothetical budget line is given by the budget $\left\{\mathbf{p}_{0}, x_{0}\right\}$. The points at which the two expansion paths intersect the new budget, at $\mathbf{q}_{1}\left(\tilde{x}_{1}\right)$ and $\mathbf{q}_{2}\left(\tilde{x}_{2}\right)$, are termed intersection demands. ${ }^{2}$ Formally, the $\tilde{x}_{t}$ at which expansion path $\mathbf{q}_{1}(x)$ intersects is defined implicitly by the equation:

$$
\mathbf{p}_{0}^{\prime} \mathbf{q}_{0}=\mathbf{p}_{0}^{\prime} \mathbf{q}_{t}\left(\tilde{x}_{t}\right)
$$

Given uniqueness and normality, a unique value of $\tilde{x}_{t}$ will always exist for every expansion path. The budget lines for the intersection demands are labelled $\left\{\mathbf{p}_{1}, \widetilde{x}_{1}\right\}$ and $\left\{\mathbf{p}_{2}, \widetilde{x}_{2}\right\}$ respectively. Given the budget lines we have drawn, the two intersection demands satisfy SARP since neither is revealed weakly preferred to the other. Given all this, we are now in a position to identify the points on the $\left\{\mathbf{p}_{0}, x_{0}\right\}$ budget line that are consistent with utility theory and the observed expansion paths. These are simply the points $\mathbf{q}_{0}$ such that $\left\{\left(\mathbf{p}_{0}, \mathbf{q}_{0}\right),\left(\mathbf{p}_{1}, \mathbf{q}_{1}\right),\left(\mathbf{p}_{2}, \mathbf{q}_{2}\right)\right\}$ satisfy SARP. The set of all such points are labelled $S\left(\mathbf{p}_{0}, x_{0}\right) ;$ following Varian (1982) we term this the support set for $\left\{\mathbf{p}_{0}, x_{0}\right\}$. It will be clear that any point on the new budget line that is not in $S\left(\mathbf{p}_{0}, x_{0}\right)$ leads to a violation of SARP. Conversely, any point in $S\left(\mathbf{p}_{0}, x_{0}\right)$ is weakly revealed preferred to the intersection demands and they are not directly revealed preferred to any point in the interior of $S\left(\mathbf{p}_{0}, x_{0}\right)$. Extending this insight to many goods and many periods we have the following general definition for the support set for any $\left(\mathbf{p}_{0}, x_{0}\right)$ :

$$
S\left(\mathbf{p}_{0}, x_{0}\right)=\left\{\begin{array}{c}
\mathbf{q}_{0} \geq \mathbf{0}, \mathbf{p}_{0}^{\prime} \mathbf{q}_{0}=\mathbf{x}_{0}: \\
\left.\mathbf{q}_{0}: \mathbf{p}_{0}, \mathbf{p}_{t} ; \mathbf{q}_{0}, \mathbf{q}_{t}\left(\tilde{x}_{t}\right)\right\}_{t=1, \ldots, T} \text { satisfy SARP }
\end{array}\right\}
$$

where $\tilde{x}_{t}$ is defined as in equation (1). Note that the support set is empty if and only if the observed data set fails SARP and it is a singleton if and only if the hypothetical relative prices are the same as an observed set of relative prices; that is, if $\mathbf{p}_{0}=\lambda \mathbf{p}_{t}$ for some $t$ and $\lambda>0$. We refer to the boundaries of the support set as E-bounds since they are the bounds derived from the expansion paths. The definition of $S\left(\mathbf{p}_{0}, x_{0}\right)$ as given in (2) is not suited to

\footnotetext{
${ }^{1}$ This figure reproduces a figure in Blundell et al (2007).

${ }^{2}$ We could denote these by $\mathbf{q}_{t}\left(\tilde{x}_{t}\left(\mathbf{p}_{0}, x_{0}\right)\right)$ to emphasise the dependence of $\tilde{x}_{t}$ on the hypothetical budget but we prefer to use the clearer notation.
} 
empirical implementation as it stands; Blundell et al (2007) show how to calculate E-bounds using standard linear programming techniques.

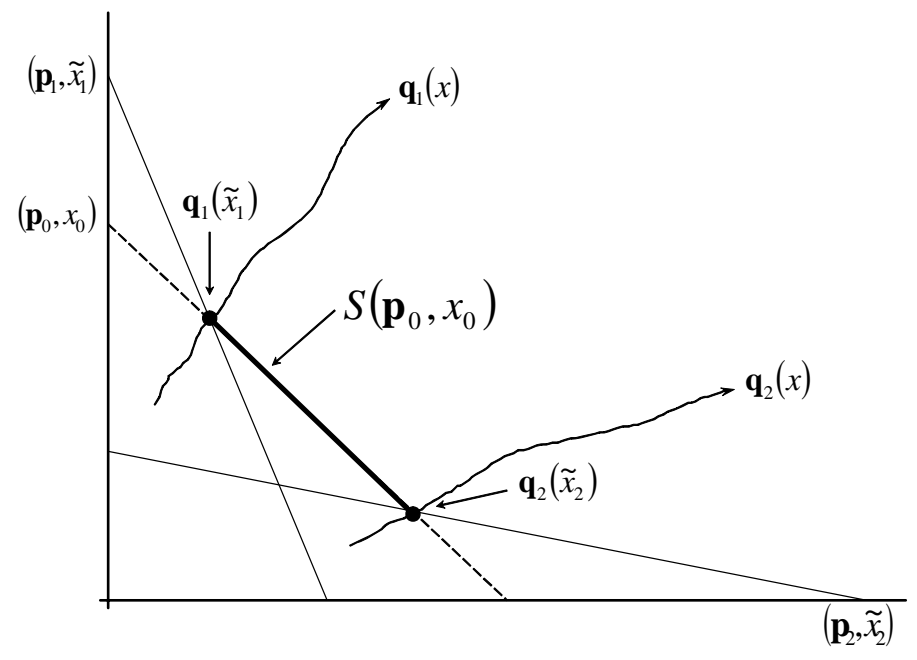

Figure 1: Defining the support set

Blundell et al (2007) show that the support set as defined here makes the best use of the data in the following sense. Take any points on the expansion paths, $\hat{\mathbf{q}}_{t}$ for $t=1, . . T$ and construct the pseudo-support set

$$
S^{\prime}\left(\mathbf{p}_{0}, x_{0}\right)=\left\{\mathbf{q}_{0}: \begin{array}{c}
\mathbf{q}_{0} \geq \mathbf{0}, \mathbf{p}_{0}^{\prime} \mathbf{q}_{0}=\mathbf{x}_{0} \\
\left\{\mathbf{p}_{0}, \mathbf{p}_{t} ; \mathbf{q}_{0}, \hat{\mathbf{q}}_{t}\right\}_{t=1, \ldots, T} \text { satisfy SARP }
\end{array}\right\}
$$

Blundell et al (2007) show that $S^{\prime}\left(\mathbf{p}_{0}, x_{0}\right)$ contains $S\left(\mathbf{p}_{0}, x_{0}\right)$. In general, the set inclusion will be strict if $\hat{\mathbf{q}}_{t} \neq \mathbf{q}_{t}\left(\tilde{x}_{t}\right)$ so our E-bounds are strictly smaller than those given by any other choice of observed demands to generate the support set.

With no further structural restrictions and no more information this is the most that we can squeeze out of the data and basic theory to bound demands. Considering more time periods, Blundell et al (2007) give conditions under which an extra observation $\left\{\mathbf{p}_{T+1}, \mathbf{q}_{T+1}(x)\right\}$ leads to tighter bounds. They show that adding more information in the form of a previously unobserved price vector and a corresponding expansion path is not always informative: it will only improve the bound if the new budget plane defined by the new intersection demand cuts through the original support set. The purpose of this paper is to consider how far we can improve bounds by imposing structure in form of separability restrictions. 


\section{Improving Bounds with Separability and Price Ag- gregation}

The aggregation of goods or the incorporation of some form of separability assumption is commonplace in empirical analysis of consumer behaviour. The main benefits are those brought about by the corresponding dimension reduction in the demand system and the consequent easing of the data requirements and the simplification of the estimation procedure. Here we examine the impact on bounds on demand responses that arises from assuming separability of different forms, as well as the effect of aggregating commodities.

Suppose that we are principally interested in the own-price demand curve of a particular good of interest. Partition the set of goods into the good of interest $q_{t}^{1}$ and all other goods which are labelled $\mathbf{q}_{t}^{2}$ for convenience. The utility function $u($.$) is (weakly) separable with$ respect to the group $\mathbf{q}_{t}^{2}$ if we can write the utility function as

$$
u=u\left(q_{t}^{1}, U^{2}\left(\mathbf{q}_{t}^{2}\right)\right)
$$

where $U^{2}($.$) is a sub-utility function and u$ is strictly increasing in $U^{2}$. The RP conditions for separability provided by Matzkin and Richter (1991) and Varian (1983) can be used to define a new, separability-consistent support set which we denote by $S^{S}\left(\mathbf{p}_{0}, x_{0}\right)$ :

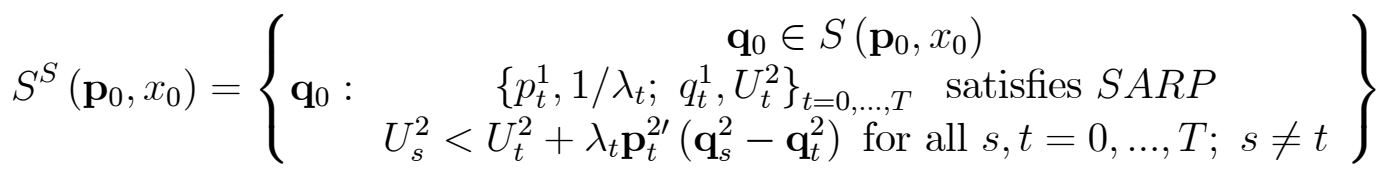

where we note that $1 / \lambda_{t}$ can be interpreted as the price index for $U_{t}^{2}$. The definition of the separability-consistent support set makes clear that the restrictions required by separability (weakly) improve the bound.

Under weak separability the price index for $\mathbf{q}_{t}^{2}$ depends on group expenditure $\left(\mathbf{p}_{t}^{2 \prime} \mathbf{q}_{t}^{2}\right)$ but we can add the requirement that $U^{2}($.$) is homothetic and define a homothetic separability-$ consistent support set which we denote by $S^{H}\left(\mathbf{p}_{0}, x_{0}\right)$

$$
S^{H}\left(\mathbf{p}_{0}, x_{0}\right)=\left\{\mathbf{q}_{0}: \mathbf{q}_{0} \in S^{S}\left(\mathbf{p}_{0}, x_{0}\right) ; U_{t}^{2} / \lambda_{t}=\mathbf{p}_{t}^{2 \prime} \mathbf{q}_{t}^{2}\right\}
$$

This further restriction weakly improves the separability-consistent bound. As well as potentially improving the bounds, the homothetic separability requirement is also computa- 
tionally convenient because it reduces the number of 'free parameters' (the Afriat Numbers $\left\{U_{t}^{2}, 1 / \lambda_{t}\right\}_{t=0, \ldots, T}$ and the demand vector $\left.\mathbf{q}_{0}\right)$ in the problem to $J+T+1$ compared to $J+2(T+1)$ in the separable case. Additively separability can also be used ${ }^{3}$ to define improved bounds using the restrictions in Varian, (1983, Theorem 6).

The use of separability restrictions improves bounds by restricting substitution responses and achieving a dimension reduction to a two group demand system (the good of interest, and "other goods"). The principal difference from a simple two-good system is that the quantity and price indices for the separable group are not known; they are represented by the Afriat Numbers $\left\{U_{t}^{2}, 1 / \lambda_{t}\right\}_{t=0, \ldots, T}$ and whilst their values are restricted by the inequalities in (5) and (6), any values which satisfy these requirements are valid. Since the support set will, in general, depend on the selected values for these Afriat Numbers, it needs to be defined over all admissible values. If, however, the price and quantities indices were known uniquely, then the support set could be defined for the two-good system using (2) with the price and quantity indices replacing the data for the group.

The impact of utilising these restrictions can be seen from the Figure 2. This simulation utilises Cobb-Douglas preferences over three goods with four observed relative price regimes. The simulated data is homothetically separable for any two of the three goods and the true demand curve for the remaining good (the good of interest) is illustrated. We then consider the gains of various improvements to the basic support set. The bounds labelled "RP Bounds" illustrate the maximum and minimum demands for the good of interest within the support set defined in (2). As expected, these bounds are the widest and we note first the improvement from exploiting homothetic separability restrictions (labelled "RP and Separability Bounds") and then the further improvement which can be made if we use the correct Cobb-Douglas price and quantities indices for the separable group (labelled RP and Index Number Aggregation") thereby reducing the dimension of the problem to a simple two-good situation.

\footnotetext{
${ }^{3}$ We are grateful to a referee for pointing this out.
} 


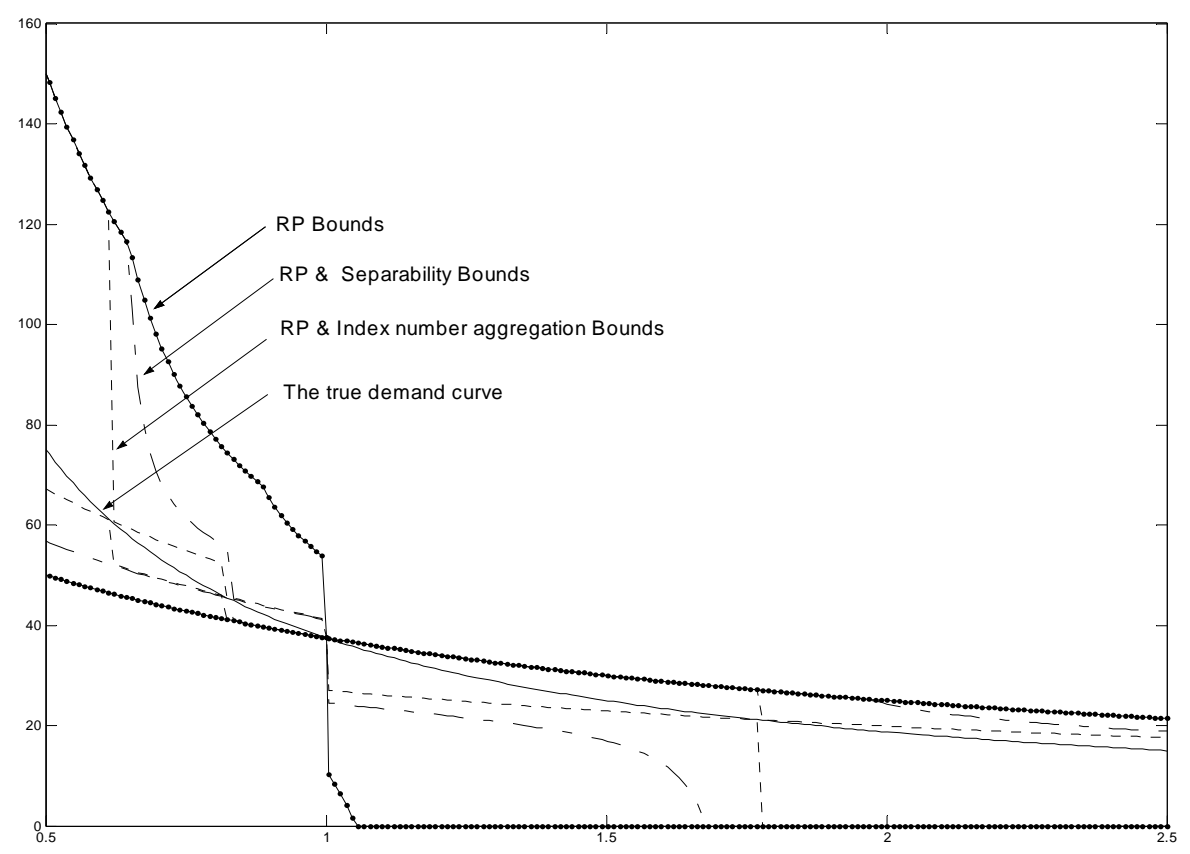

Figure 2: Improving E-Bounds under Separability and Aggregation

\section{An Empirical Analysis of Demand Bound Improve- ments}

\subsection{Data}

In this analysis we begin by taking three broad consumption goods: food, other nondurables, and services (for precise definitions see the Appendix). These are the same commodity groupings used in Blundell et al (2007). The empirical analysis uses the same 25 years of British Family Expenditure Surveys from 1975 to 1999. This choice is made because in many contexts the price responsiveness of food relative to services and to other nondurables is of particular interest. It is a key parameter in the indirect tax debate. Although food is largely free of value added tax (VAT) in the UK, the discussions over the harmonisation of indirect tax rates across Europe and the implications of a flat expenditure tax raised uniformly across all consumption items requires a good understanding of food demand responses across the income distribution. It is also important in general discussions of cost of living changes across the income distribution. Relative food prices saw some abrupt rises as the tariff structure and 
food import quotas were changed in Europe early in the period under study. In our discussion of separability we also examine a higher dimensional system. The Family Expenditure Survey is a repeated cross-section survey consisting of around 7,000 households in each year. From these data we draw a relatively homogeneous sub-sample of couples with children. This gives us between 1,421 and 1,906 observations per year and 40,731 observations over the entire period. We use total spending on nondurables to define our total expenditure variable. Figure 3 shows the mean budget shares for these goods over the period ${ }^{4}$. As can be seen, the mean budget share for food exhibits a large fall whereas services are rising steadily over our data period.

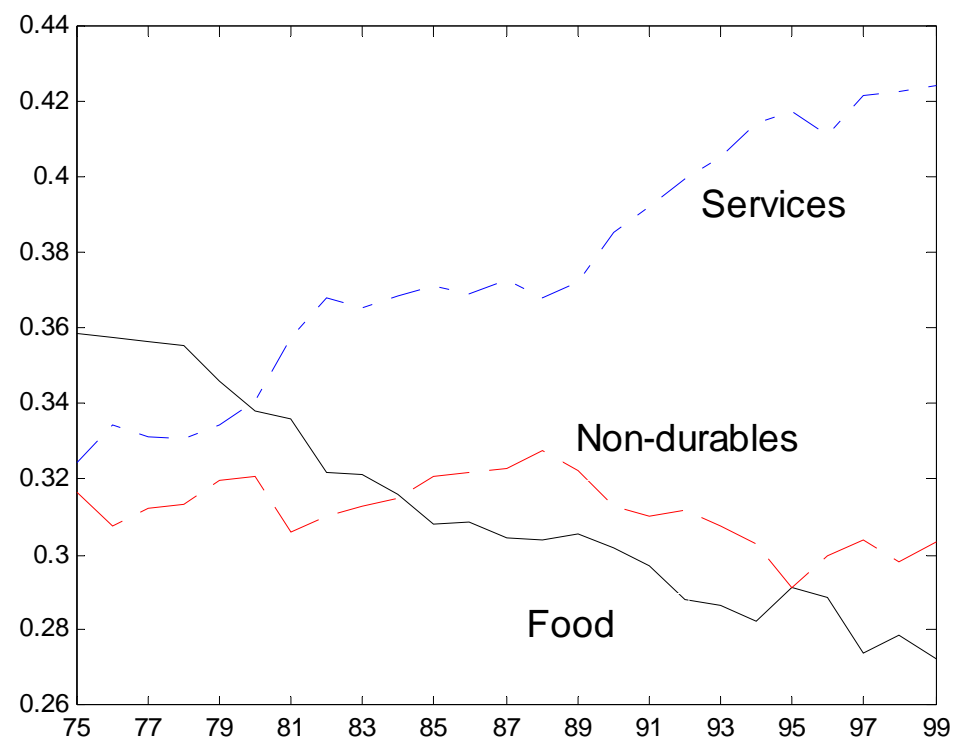

Figure 3: Mean budget shares.

The substantial relative price variation can be seen in the dated points in Figure 4. This figure shows the scatter plot of the prices of food and services relative to nondurables. The relative prices show a dramatic change in the mid to late-1970's. The figure also illustrates the convex hull of the relative price data. The annual price indices for these commodity groups are taken from the annual Retail Prices Index. Nondurables are treated as the numeraire good. We see a steadily rising price for services relative to food and nondurables.

\footnotetext{
${ }^{4}$ Further details are provided in Blundell, et al (2007).
} 


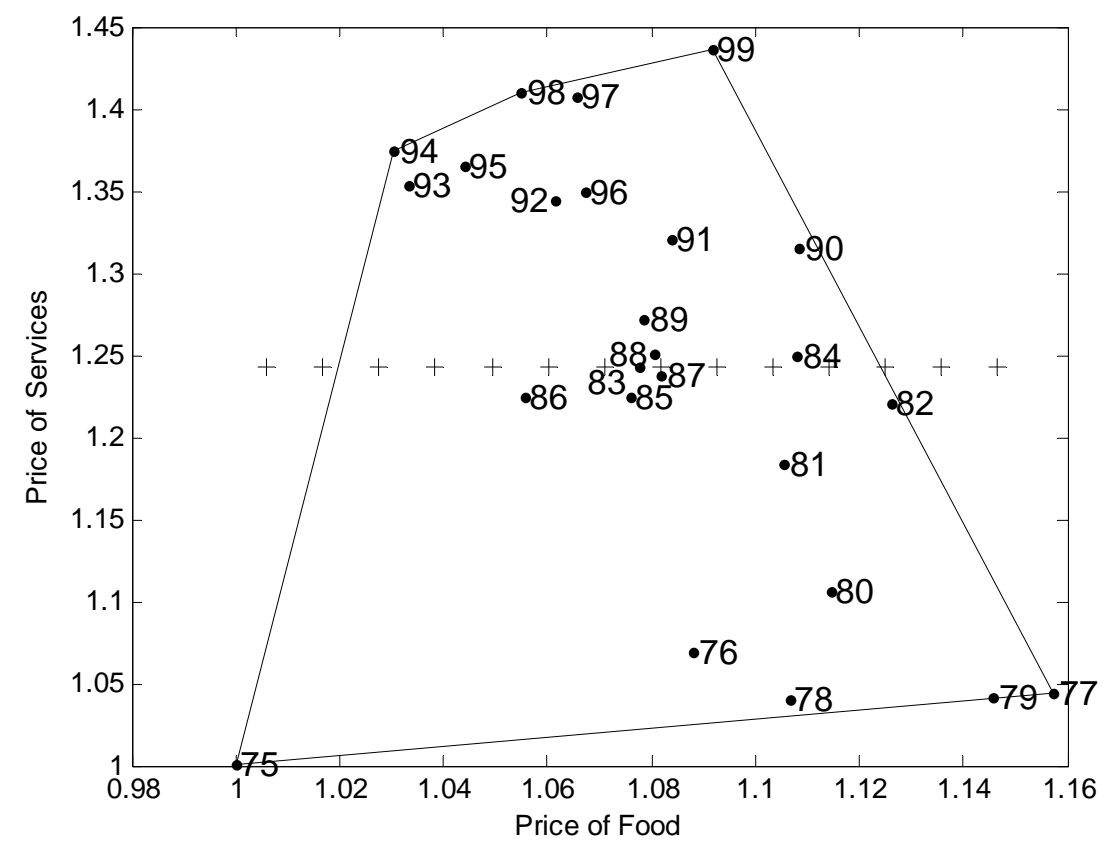

Figure 4: Relative prices, 1975 - 1999.

\subsection{Estimating Expansion Paths}

Consumers who are observed in the same time period and location are assumed to face the same relative prices. Under this assumption, Engel curves for each location and period correspond to expansion paths for each price regime. we use a shape invariant specification to pool over different demographic types of households (see, for example, Blundell, Browning and Crawford (2003) who show that is an effective way of allowing for demographics). We account for the possible endogeneity of total expenditure using the control function approach (see Blundell and Powell $(2003)) \cdot{ }^{5}$ Let $\mathbf{d}^{i}$ represent a $(D \times 1)$ vector of household composition variables relating to household $i$. Our specification takes the form:

$$
w_{j}^{i}=g_{j}\left(\ln x_{i}-\phi\left(\mathbf{d}_{i}^{\prime} \boldsymbol{\alpha}\right)\right)+\mathbf{d}_{i}^{\prime} \gamma_{j}+\varepsilon_{j}^{i}
$$

where $w_{j}^{i}$ is the expenditure share for household $i$ on good $j$. To account for the endogeneity of $\ln x$ we specify:

$$
\ln x_{i}=\mathbf{z}_{i}^{\prime} \pi+v_{i}
$$

\footnotetext{
${ }^{5}$ This is analysed in Blundell, Chen and Kristensen (2003) and compared to a the fully nonparametric instrument variables (NPIV) case. It is found to account quite well for the endogeneity of total expenditure in comparison to a full NPIV approach.
} 
where $\mathbf{z}$ are a set of variables which include the demographic variables $\mathbf{d}_{i}$ and earned income as an excluded instrument. The required assumption for the control function approach is:

$$
E\left(\varepsilon_{j}^{i} \mid \ln x_{i}, \mathbf{d}_{i}, v_{i}\right)=0
$$

so that semiparametric regression using an augmented equation (7) that includes functions of $v_{i}$ will produce consistent estimates of $g_{j}, \boldsymbol{\alpha}$ and $\boldsymbol{\gamma}$ (see Newey, Powell and Vella (1999)).

\subsection{E-Bounds on Demand Responses}

We begin by illustrating the idea of E-bounds using a three good demand system. Using the estimated expansion paths we recover the intersection demands for each $\left\{\mathbf{p}_{0}, x_{0}\right\}$ budget and check the revealed preference conditions for the data $\left\{\mathbf{p}_{t}, \mathbf{q}_{t}\left(\tilde{x}_{t}\right)\right\}$. We find (by searching for chronologically ordered SARP-consistent sub-periods) that the periods 1982 to 1991 satisfy SARP. Figure 5 shows the convex hull of the price data corresponding to this sub-period. Comparison of Figures 4 and 5 shows the reduction in the space spanned by the convex hull of the prices once SARP-violating intersection demands have been dropped.

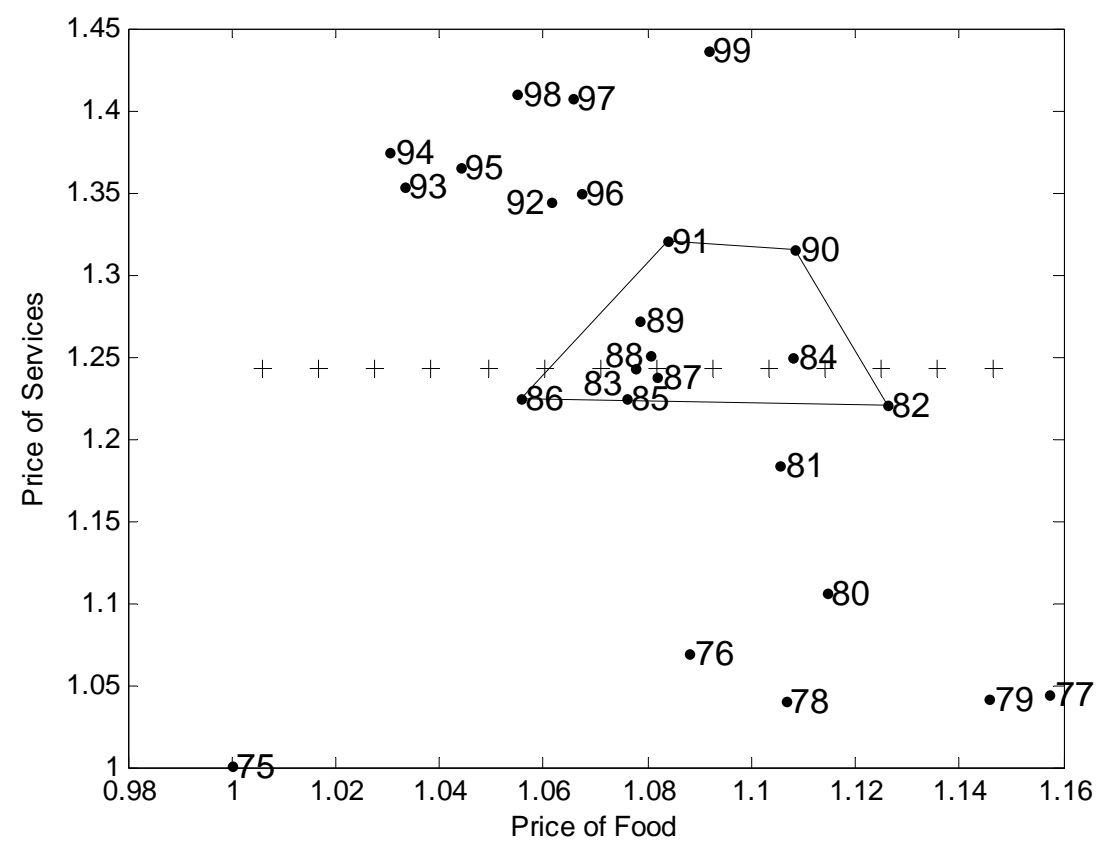

Figure 5: Price scatter plot of the SARP-consistent intersection demands 
In Figure 6, 7 and $8^{6}$ we present the own and cross-price demand curves for food for the median income individual using the reduced set of SARP-consistent observations. As can be seen from a comparison with Figures 4, the bounds on the food demand curve are particularly tight when the $\mathbf{p}_{0}$ vector is in the dense part of the observed price data. Outside the convex hull of the price data the E-Bounds widen considerably; so much so, indeed, that we cannot rule out that households would stop buying food if the relative price of food rose a little above the price seen in 1982! We now consider whether imposing separability tightens the bounds.

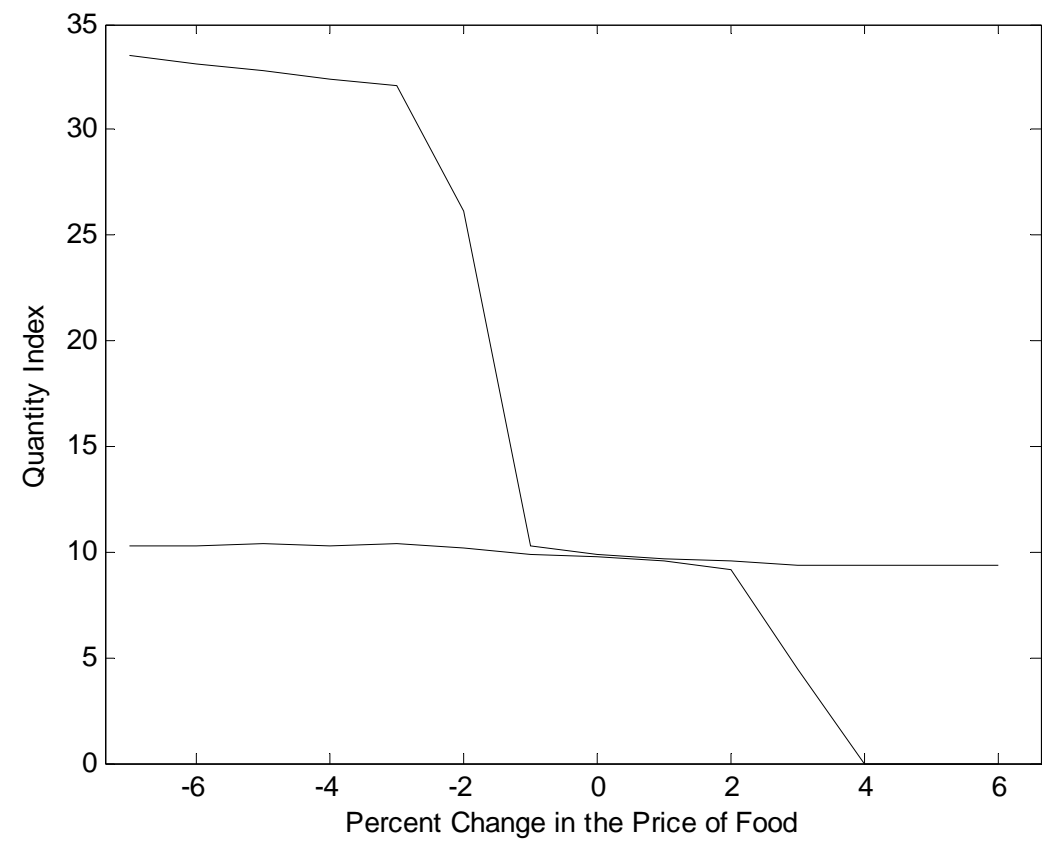

Figure 6: The own-price demand bounds for Food

\subsection{E-Bound Improvements with Separability}

We begin the analysis of the improvements afforded by adding separability restrictions to those which just use the intersection demands and SARP by looking at how the bounds on the own demand curve for Food change when nondurables and services are grouped together in a separable subgroup. The raw data reject weak separability of services and nondurables with respect to food. We have therefore imposed separability at the intersection demands using the minimum distance criteria developed in Blundell, Browning and Crawford (2007),

\footnotetext{
${ }^{6}$ These figures reproduce figures in Blundell et al (2007).
} 


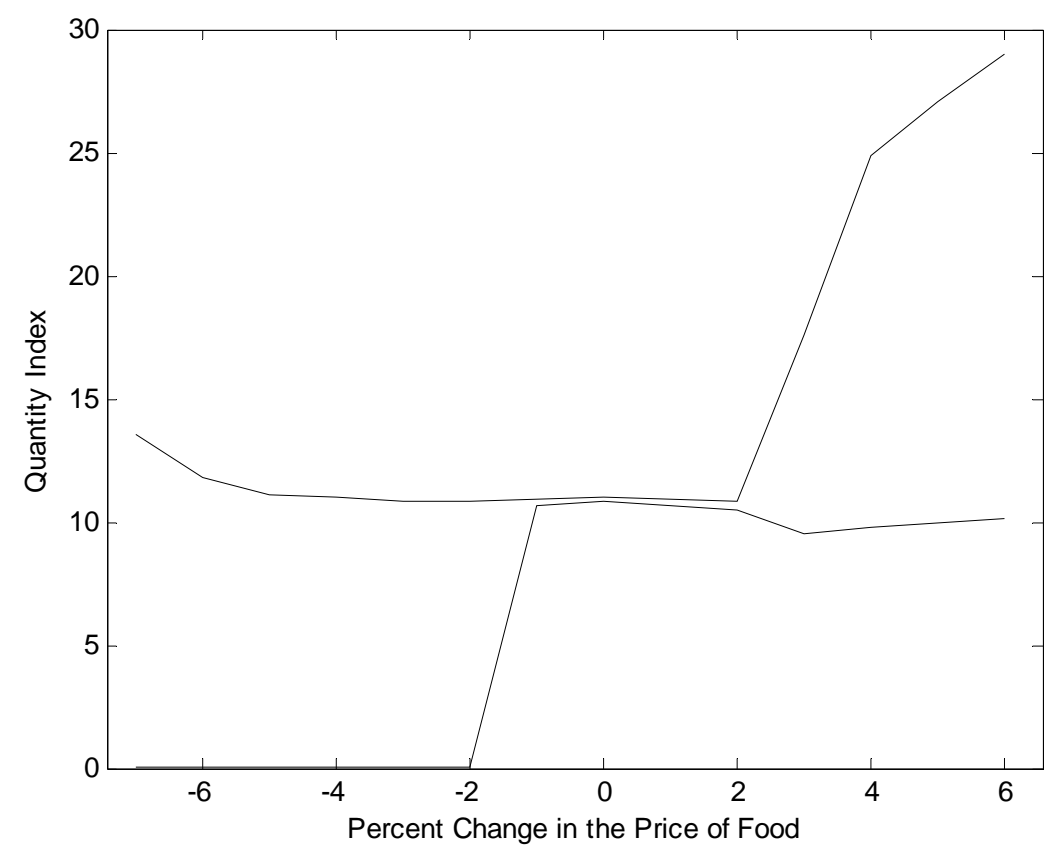

Figure 7: The cross-price demand bounds for Non-durables

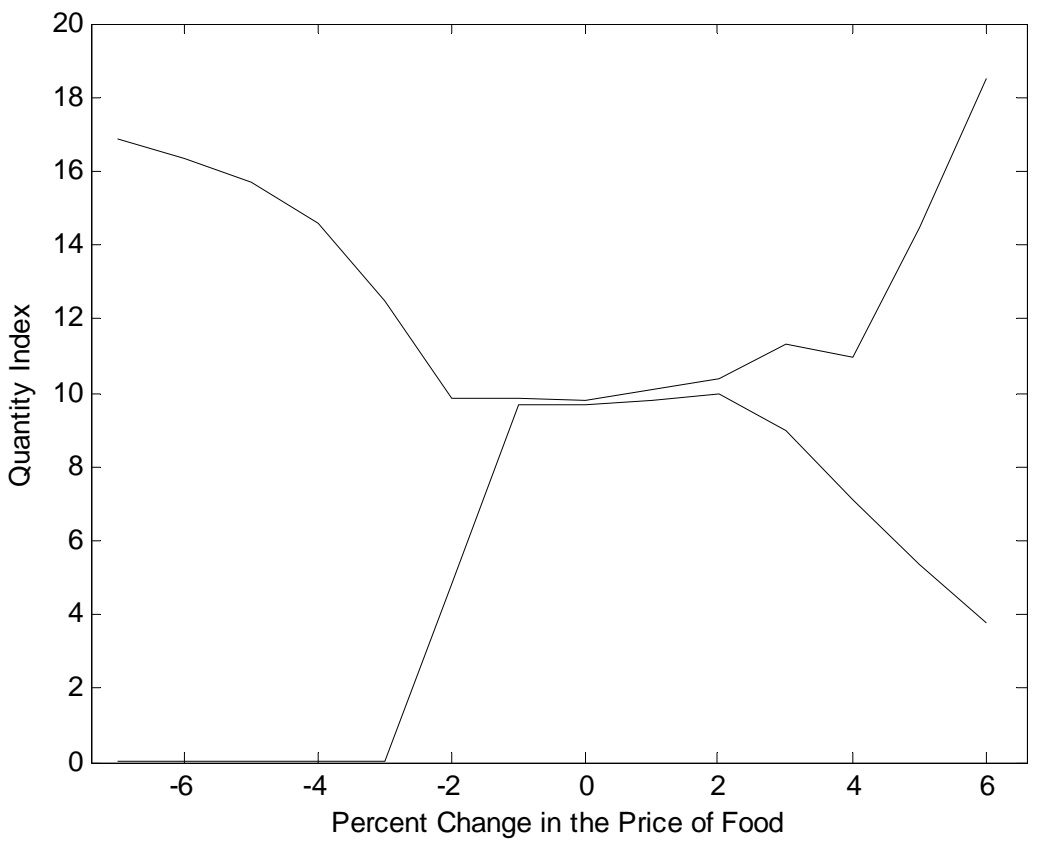

Figure 8: The cross-price demand bounds for Services 
suitably augmented to account for separability restrictions. The intersection demands have thus been perturbed by the minimum (Euclidean) distance such that they satisfy the RP conditions for separability provided by Matzkin and Richter (1991) and Varian (1983). Using the separability-consistent intersection demands we have computed the support sets (2) and (5) and the resulting own-price and cross-price bounds are illustrated in Figures 9, 10 and 11. The bounds with the separability restrictions are shown as dashed lines and the bounds without separability are shown by the solid lines (the same as in Figures 6-8). Note that since we use 'perturbed intersection points, the bounds with separability imposed are not always inside the unrestricted bounds, but they are very close.

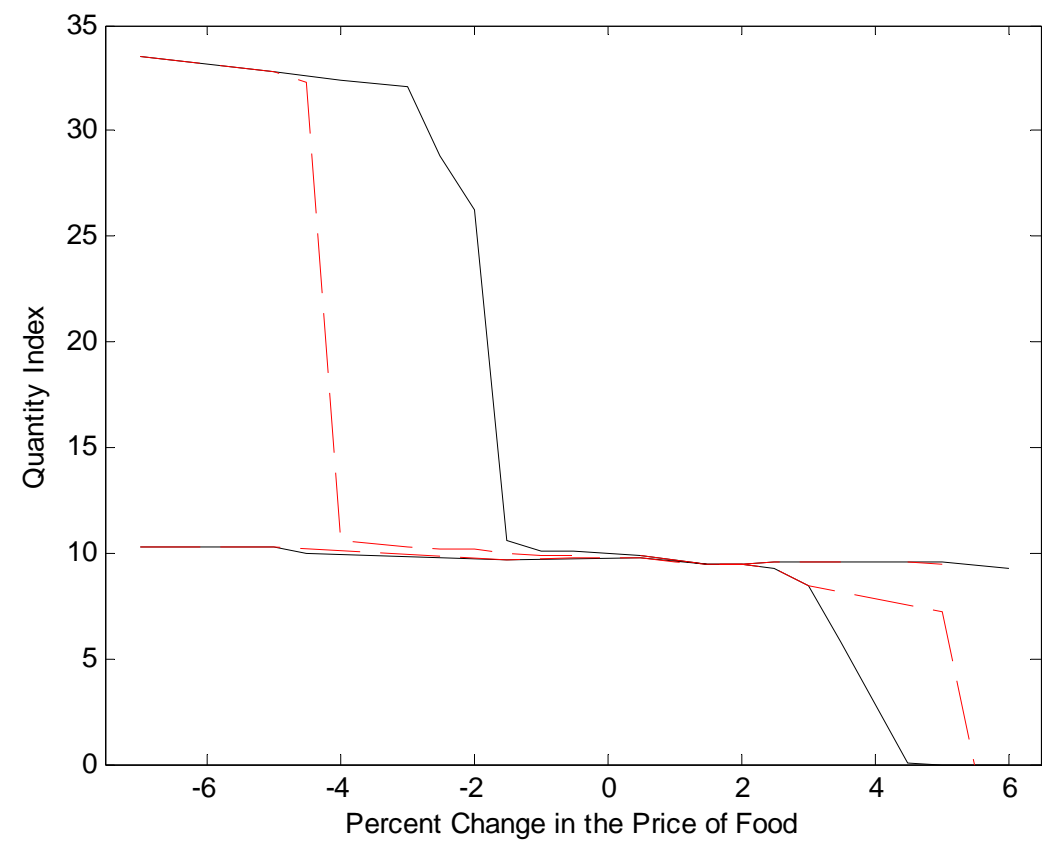

Figure 9: The own-price demand bounds for Food - with and without separability restrictions

The general improvement in the bounds which separability restrictions provide is immediately apparent in the figures. This is particularly so when we consider the range of price variation over which it is possible to find tight bounds. Previously we could only be reasonably precise over the range $[-1.8 \%$ to $+2.5 \%]$ of the mean price of food. The use of separability restrictions expands this to approximately [-4\% to $+5 \%]$. What is also apparent, however, is that within ranges where the demand curve bounds where already tight, the 


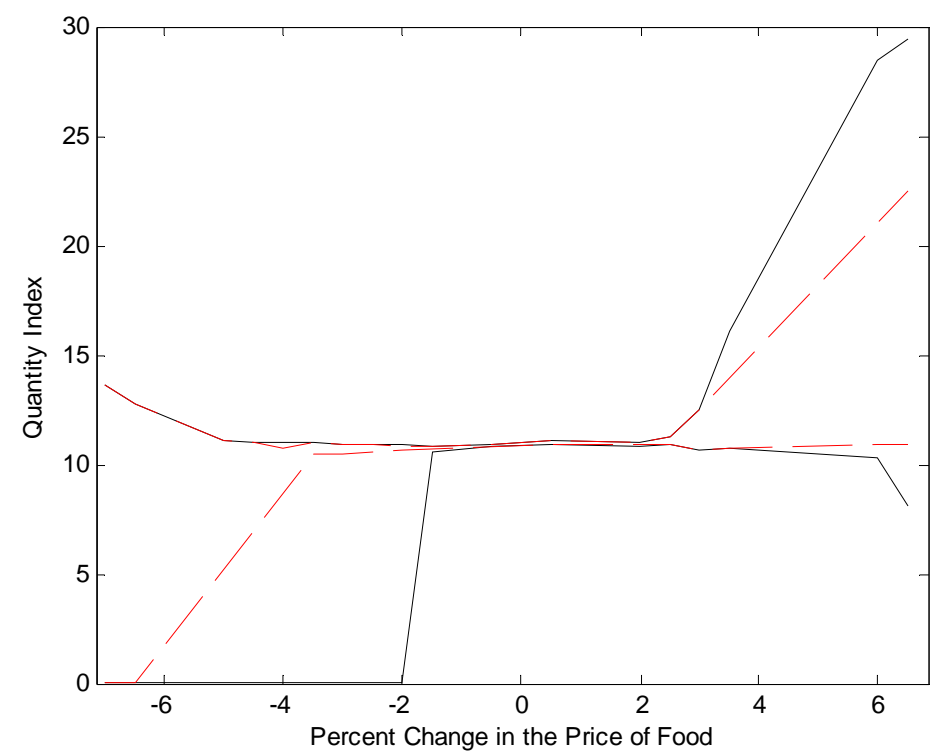

Figure 10: The cross-price demand bounds for Non-durables - with and without separability restrictions

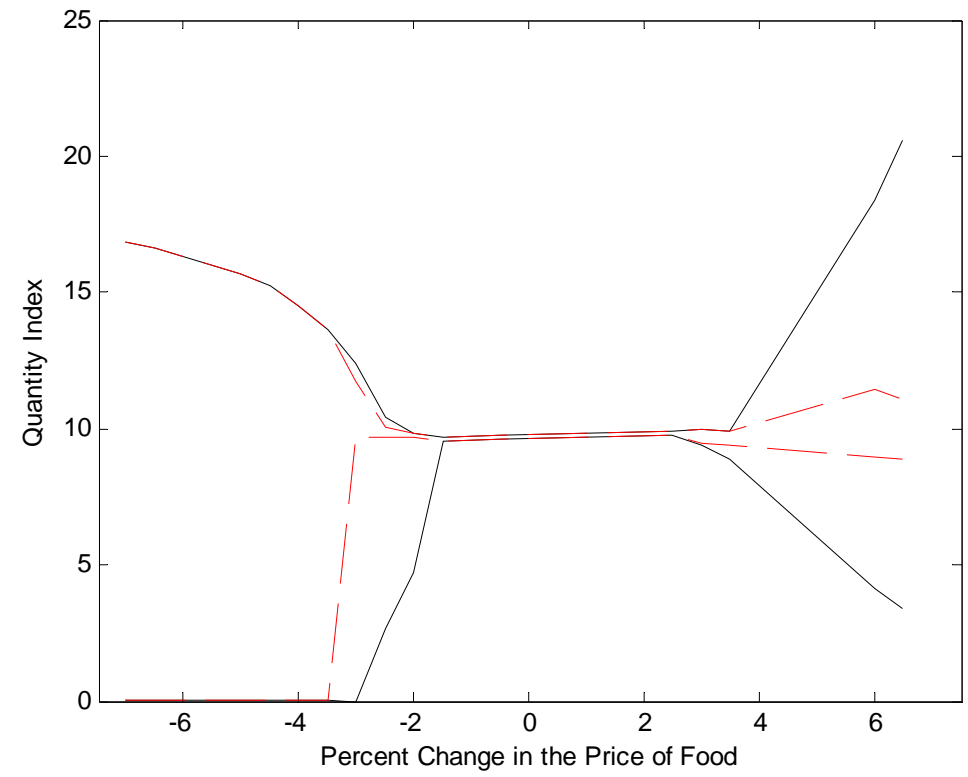

Figure 11: The cross-price demand bounds for Services - with and without separability restrictions 
improvement brought about by the separability restrictions is relatively modest. Of course, separability restrictions come into their own in demand systems which need to accommodate a larger number of goods. The greater the level of disaggregation confronting the researcher, the greater the potential benefits of separability. It is to this issue of separability in higher dimensional restrictions which we now turn.

\subsection{E-Bound Improvements in Higher dimensional systems with Separability}

In this section we consider the impact of imposing separability restrictions on the E-bounds on demand responses in a higher dimensional case in which preferences over many goods are constrained by separability restrictions over subsets of these goods. In particular, we take an expanded set of demands over six commodity groups which break up the food, other goods and services into six categories, food consumed at home ("Food In"), food consumed away from home ("Food Out"), alcohol and tobacco, other nondurables, services and travel (see Appendix for the breakdown of the commodity groups). We examine the two food categories and consider separability restrictions on the other goods.

It might be expected that the real improvements in the E-bounds from separability come from reducing a higher dimensional problem. Indeed the gains could be substantial when there are many goods. The changes in VAT rates over the period provide useful variation in the relative price of food consumed inside (which is not eligible for VAT) and food consumed outside (which is eligible). In the following examples we continue to use the sub-period 1982 through 1991.

Figure 12 shows the own-price demand curve for food consumed at home (Food In) in the 6 good system (outer solid lines). The first obvious effect is that of the impact of disaggregation on the bounds: compared to those in the previous section the demand curve bounds are far wider reflecting perhaps the more general nature of the substitution possibilities in a six good system compared to a three good system. We then use the separability restriction on the "other goods" (alcohol and tobacco, other nondurables, services and travel) and recompute the E-bounds focussing on the own price response of Food In and the cross price effects on the demand for food consumed outside the home. The results are shown by the inner dashed 


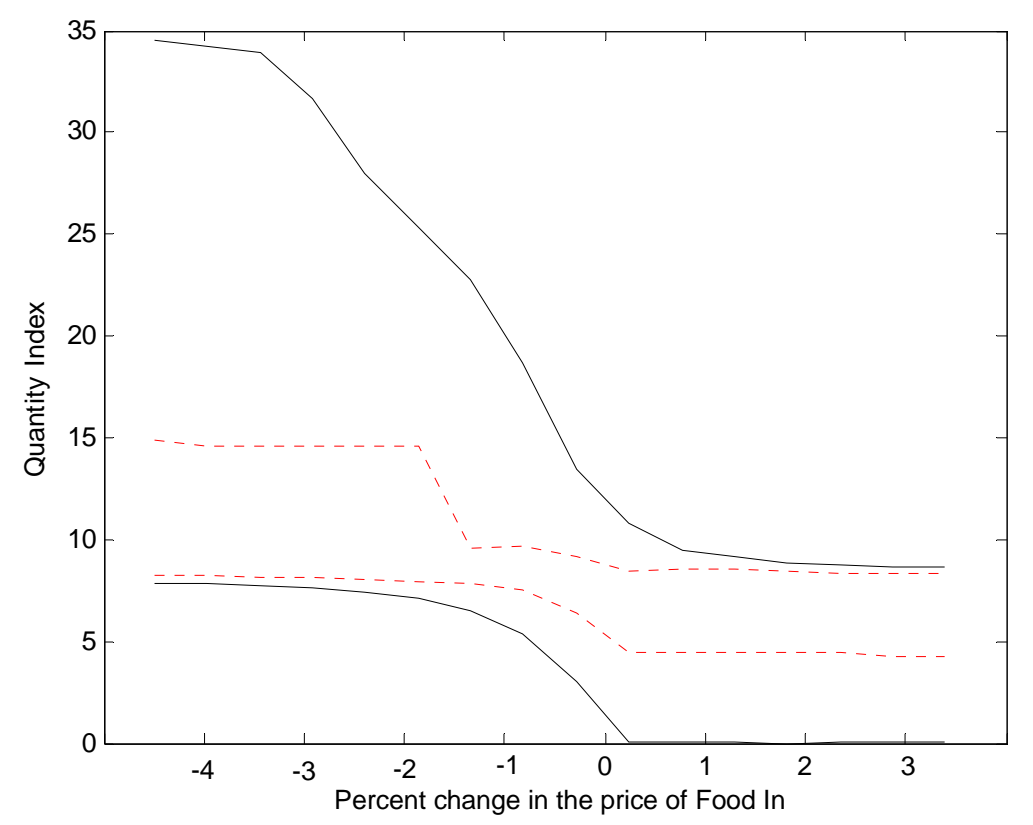

Figure 12: Separability in a Higher Demension System - The own price demand bounds for Food In

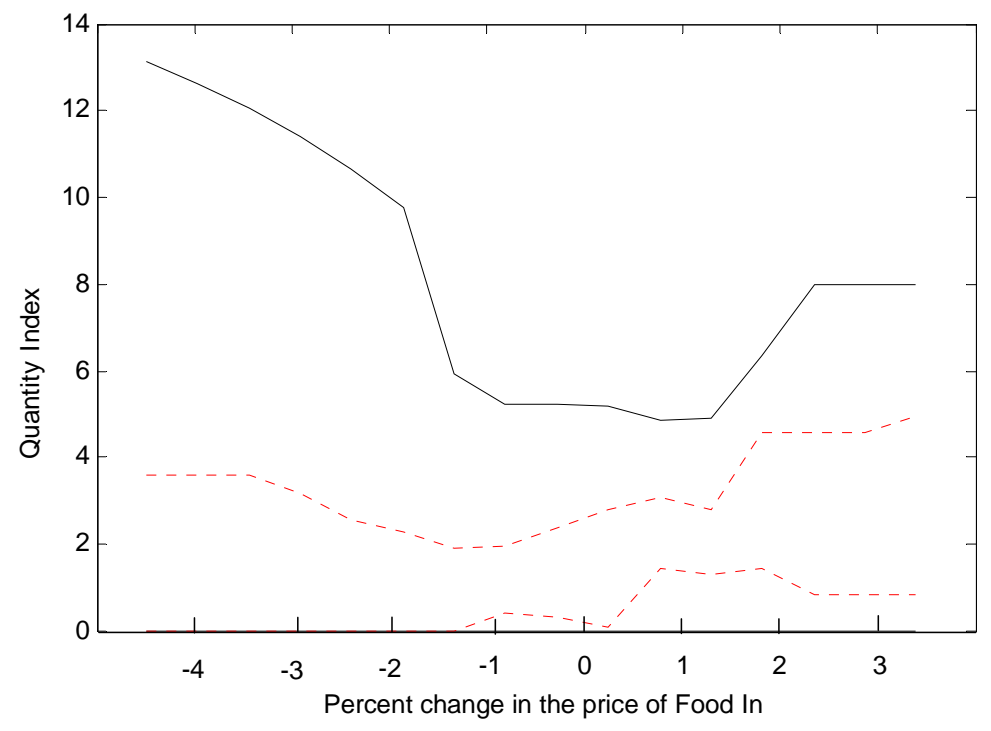

Figure 13: Separability in a Higher Demension System - The own price demand bounds for Food In 
bounds. A large improvement in the bounds is apparent but note that the resulting bounds form this separable system are wider than those for the three good system because of the flexibility over the choice of Afriat numbers compared to simple aggregation - precisely as one would expect given the discussion around Figure 2. Figure 13 show that the gains are equally large for the cross-price effect on food consumed outside the home.

\section{Summary and Conclusions}

The research reported in this paper forms part of a broad agenda examining key dimensions by which revealed preference bounds on consumer demand responses to price variation can be improved. In earlier research we have shown how improvements can arise from using expansion paths for given relative prices, E-bounds. We have also documented conditions under which new price information improves these E-bounds. In this paper we have considered the way in which revealed preference bounds on consumer demand responses to price variation can be improved using separability assumptions. We have also shown how to impose these separability assumptions within a fully nonparametric analysis and distinguish between weak, homothetic and exact index separability.

Looking forward, we would like to examine the distribution of demands across the heterogeneity distribution and not focus on average demands at all, see Brown and Matzkin (1998). If we describe consumer behaviour by the share equations $\mathbf{w}=\mathbf{g}(\ln x, \ln \mathbf{p}, \mathbf{d}, \boldsymbol{\varepsilon})$ where $\boldsymbol{\varepsilon}$ is a $J-1$ vector of unobservable heterogeneity then global invertibility is required to identify the complete distribution of demands (see Brown and Matzkin (1998) and Beckert and Blundell (2005)). In this case generalisations of quantile regression are required for estimation, see Matzkin (2006). For the more limited case considered here we could ask whether there is a general interpretation of local average demands that is consistent with consumer behaviour. For empirical demand analysis a natural assumption is that unobservable preference heterogeneity, conditional on observable demographics, is independent of prices and total outlay: $F(\varepsilon \mid \ln x, \ln \mathbf{p}, \mathbf{d})=F(\varepsilon \mid \mathbf{d})$. The covariance between budget shares and the responsiveness of these to changes in log total outlay, conditional on the observable determinants of demand, 
is defined as

$$
H(\ln x, \ln \mathbf{p}, \mathbf{d})=\operatorname{cov}\left(\frac{\partial \mathbf{g}}{\partial \ln x}, \mathbf{g}^{\prime} \mid \ln x, \ln \mathbf{p}, \mathbf{d}\right)
$$

Lewbel (2001) shows that average demands of rational consumers satisfy integrability conditions iff $H($.$) is symmetric and positive semidefinite. { }^{7}$ If $H$ is small relative to the the Slutsky matrix for these average demands, then the system will be 'close' to integrable. This result extends the usefulness of the local average regression results used in this paper in the presence of nonseparable unobserved heterogeneity. However, as stressed above, in future research we will want to describe and predict the complete distribution of demands.

\footnotetext{
${ }^{7}$ For example, in the Almost Ideal Demand system (Deaton and Muellbuaer, 1980), heterogeneity in the $a(p)$ parameters would automatically satisfy this condition.
} 


\section{References}

[1] Afriat, S.N. (1967), "On a System of Inequalities in Demand Analysis: An Extension of the Classical Method", International Economic Review, 14, 460-472.

[2] Banks, J. Blundell, R.W. and Lewbel, A. (1997), "Quadratic Engel Curves, Indirect Tax Reform and Welfare Measurement", Review of Economics and Statistics, LXXIX, 4, $527-539$.

[3] Beckert, W. and R.W. Blundell (2005), "Invertibility of Nonparametric Stochastic Demand Functions", Cemmap Working Paper CWP09/05, July.

[4] Blundell, R., M. Browning and I. Crawford (2003), "Nonparametric Engel Curves and Revealed Preference" Econometrica, Vol.71, No.1. January, 205-240.

[5] Blundell, R., M. Browning and I. Crawford (2007), "Best Nonparametric Bounds on Demand Responses", Walras-Bowley Lecture, IFS Working Paper, 05/20, revised February 2007.

[6] Blundell, R., Chen, X. and D. Kristensen, (2003) "Semiparametric Engel Curves with Endogenous Expenditure", CeMMAP Working Paper, IFS, CWP 15/03.

[7] Blundell, R. P. Pashardes and G. Weber, (1993), "What do we Learn about Consumer Demand Patterns from Micro Data?", American Economic Review, 83, No.3, 570-597

[8] Blundell, R. and J. Powell, (2003), "Endogeneity in Nonparametric and Semiparametric Regression Models", Chapter 8 in Dewatripont, M., Hansen, L. and S. J. Turnovsky (eds), Advances in Economics and Econometrics, Cambridge University Press, ESM 36, pp 312-357.

[9] Brown, D.J. \& R.L. Matzkin (1998) "Estimation of Simultaneous Equations Models, with an Application to Consumer Demand" Cowles Foundation Discussion Paper.

[10] Deaton A. S. and J. Muellbauer (1980), "An Almost Ideal Demand System", American Economic Review, 70, 312-36.

[11] Hausman, J. A., Newey, W. K., Ichimura, H. and J. L. Powell (1991), "Identification and estimation of polynomial errors-in-variables models", Journal of Econometrics, 50(3), 273-295

[12] Jorgenson, D.W., L.J.Lau, and T.M.Stoker (1982), "The Transcendental Logarithmic Model of Aggregate Consumer Behavior", in Advances in Econometrics, Vol1, ed. by R. Basmann and G. Rhodes, Greenwich, Connecticut: JAI Press.

[13] Lewbel, A. (1991), "The rank of demand systems: theory and nonparametric estimation", Econometrica, 59, 711-730. 
[14] Lewbel, A (1996), "Demand systems with and without errors: reconciling econometric, random utility and GARP models", American Economic Review.

[15] Manski, C. (2003), "Partial Identification of Probability Distributions", Springer-Verlag.

[16] Matzkin (2006), "Heterogeneous Choice", forthcoming in Blundell, R. W. Newey and T. Persson (eds), Advance in Econometrics: Proceedings of the 9th World Congress; Econometric Society Monographs, Cambridge.

[17] Matzkin, R. L and M. K. Richter, (1991), "Testing Strictly Concave Rationality", Journal of Economic Theory, 53(2), 287-303.

[18] McFadden, D.L. and K. Richter (1990): "Stochastic Rationality and Revealed Stochastic Preference", in J. Chipman, D. McFadden, K. Richter, eds.: Preference, Uncertainty, and Rationality, Westview Press, 161-186

[19] Newey, W. Powell, J. and F. Vella (1999): "Nonparametric Estimation of Triangular Simultaneous Equations Models," Econometrica, 67, 565-604.

[20] Varian, H.(1982), "The Nonparametric Approach to Demand Analysis", Econometrica, 50, 945-974.

[21] Varian, H.(1983), "Nonparametric Tests of Consumer Behaviour", Review of Economic Studies, 99-110.

[22] Varian, H.(1986), "Nonparametric Analysis of Optimizing Behavior with Measurement Error", Journal of Econometrics30, 445-459. 


\section{Appendix: Commodity Groups}

\section{6 commodity group system}

"Food In": \{bread,cereals, biscuits \& cakes, beef, lamb, pork, bacon, poultry, other meats \& fish, butter, oil \& fats, cheese, eggs, fresh milk, milk products, tea, coffee, soft drinks, sugar \& preserves, sweets \& chocolate, potatoes, other vegetables, fruit, other foods $\}$.

"Food Out": \{canteen meals, other restaurant meals and snacks\}.

"Alcohol \& tobacco": \{beer, wine \& spirits, cigarettes, other tobacco $\}$

"Other Non-durables": \{household consumables, petcare, mens outer clothes, women's outer clothes, children's outer clothes, other clothes, footwear, chemist's goods, audio visual goods, records and toys,book \& newspapers, gardening goods\}

"Services": \{domestic fuels, postage \& telephone, domestic services, fees \& subscriptions, personal services\}.

"Travel": \{maintenance of motor vehicles, petrol and oil, vehicle tax and insurance, travel fares, tv licences, entertainment\}.

\section{3 commodity group system}

"Food" = "Food In" + "Food Out"

"Non-durables" = "Alcohol \& tobacco" + "Non-durables"

"Services" = "Services" + "Travel" 